\title{
Rosa de corte mexicana: disponibilidad a pagar del consumidor y su percepción de calidad
}

\author{
Diana Ruth Nervis Quevedo \\ Universidad Autónoma del Estado de México \\ México \\ Jessica Alejandra Avitia Rodríguez \\ Universidad Autónoma del Estado de México \\ México \\ Javier Jesús Ramírez Hernández \\ México
} Centro de Estudios e Investigación en Desarrollo Sustentable 


\title{
Rosa de corte mexicana: disponibilidad a pagar del consumidor y su percepción de calidad
}

\author{
Diana Ruth Nervis Quevedo ${ }^{1}$, Jessica Alejandra Avitia Rodríguez ${ }^{(\mathbb{D})}$, y Javier Jesús \\ Ramírez Hernández ${ }^{2}$ iD \\ ${ }^{1}$ Universidad Autónoma del Estado de México, México \\ ${ }^{2}$ Centro de Estudios e Investigación en Desarrollo Sustentable, México
}

Para citaciones: Nervis, D., Avitia, J., y Ramírez, J. (2020). Rosa de corte mexicana: disponibilidad a pagar del consumidor y su percepción de calidad. Panorama Económico, 28(2), 80-93. https://doi.org/10.32997/pe$\underline{2020-2690}$

Recibido: 03/09/2019

Aprobado: 5/12/2019

Autor de correspondencia: Jessica Alejandra Avitia Rodríguez jaavitiar@uaemex.mx

Editor: Andrés Escobar Espinoza Universidad de Cartagena, Colombia

Tipología IBN Publindex: Artículo de investigación científica y tecnológica
Copyright: (C) 2020. Nervis, D., Avitia, J., y Ramírez, J. Este es un artículo de acceso abierto, distribuido bajo los términos de la licencia https://creativecommons.org/licenses/by-nc-

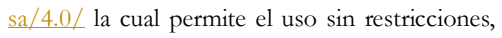
distribución y reproducción en cualquier medio, siempre y cuando que el original, el autor y la fuente sean acreditados.

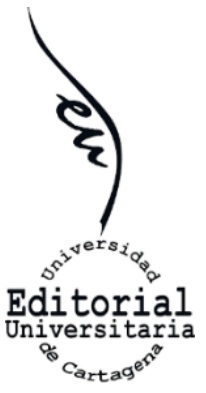

\section{RESUMEN}

La actividad florícola es una de las principales fuentes de ingresos del Sur del Estado de México, donde la cosecha de la rosa de corte es la más importante debido al valor de la producción generado. Se realiza una revisión sobre el concepto de calidad desde dos enfoques: objetivo y subjetivo; posteriormente se profundiza en los métodos no hipotéticos (subasta experimental y análisis sensorial) para determinar la disponibilidad de pago del consumidor de la rosa de corte. El objetivo de esta investigación es determinar si la percepción de calidad del consumidor influye en la disponibilidad a pagar (DAP) en el consumo de rosa de corte mexicana. En el presente estudio, la DAP de los consumidores se evalúa mediante una subasta experimental. De acuerdo con los resultados, se puede concluir que la disponibilidad a pagar (DAP) hacia la rosa de corte está vinculada a los ingresos de los consumidores y a la percepción de calidad del consumidor de la rose de corte.

Palabras clave: Disponibilidad a pagar; percepción de calidad; subasta de Vickrey; subasta experimental

JEL: C90, C91, D11, Q12

\section{ABSTRACT}

The floriculture activity is one of the main sources of revenue of the Southern region of Mexico, where the crop of the cut rose is the most relevant due to the value of the production. A review of the concept of quality is carried out from both objective and subjective approaches; subsequently, the application of non-hypothetical methods (experimental auction and sensory analysis) are used to determine the willingness to pay of consumers. The aim of this research is to determine if consumers' quality perception influences on the willingness to pay (WTP) for Mexican cut rose. Research findings suggest that consumers' willingness to pay is associated to variables such as consumers' income, and their quality perception.

Keywords: Willingness to pay; quality perception; Vickrey auctions; experimental auction.

JEL: C90, C91, D11, Q12 


\section{INTRODUCCIÓN}

La floricultura es un sector con gran potencial. Para que este se convierta en un sector económico clave para el sur del Estado de México es indispensable identificar y estimular las áreas de oportunidad. Entre ellos tenemos la promoción del producto, la difusión de una cultura sobre las flores, la distribución, alentar a los productores regionales, comerciantes y vendedores a crear asociaciones, investigaciones puntuales de mercado con el fin de conocer los mercados potenciales y consumidores finales. El Estado de México es el principal productor de flores de corte del país de México, aportando el 80 por ciento de la producción nacional (SAGARPA, 2011), donde la producción se basa en condiciones climatológicas adecuadas para la actividad florícola que le permite tener bajos costos de producción. A pesar del hecho de que la tecnología en general tiene un bajo perfil. La experiencia en la producción florícola de más de 15 años y una cultura centrada en el consumo de flores, son factores que brindan al Estado de México la oportunidad de mantener e incrementar la demanda.

El mercado del sector florícola en general es dinámico y se encuentra en constante movimiento. Los consumidores cambian sus patrones de consumo y están abiertos a nuevas variedades y colores, y están dispuestos a pagar más por ellos. Por lo tanto, existen nichos de mercado para ser explotados. Las flores son un bien suntuoso, de lujo y prescindible, por lo que su consumo está directamente relacionado con el poder adquisitivo de la población. Se estima que a medida que la economía mundial se recupere, el consumo de flores se incrementará igualmente; a medida que los países se desarrollan y alcanzan mayores estándares de vida, el consumo de flores aumentará. Debido a que las flores son un producto de lujo existe una relación directa entre el desarrollo económico del país y la demanda doméstica de flores (Gobierno de Chiapas, 2005).

Hoy en día los productores de rosa se enfrentan a la disminución de ingresos por ventas de este producto. Este problema requiere de estudiar los factores que provocan la reducción de los ingresos, entre estos factores encontramos la diferencia de percepciones de calidad de las flores entre los consumidores y los productores, la pérdida de simbolismo, la situación de compra, el precio y la preferencia por productos sustitutivos o más duraderos. El objetivo de esta investigación es determinar si la percepción de calidad del consumidor influye en la disposición a pagar en el consumo de rosa de corte. En el presente estudio, la disposición a pagar (DAP) de los consumidores se evalúa mediante una subasta experimental. Una contribución importante consiste en proporcionar más evidencia sobre la percepción de calidad de las flores de los consumidores y su relación con la DAP. Los resultados empíricos contribuyen con una literatura limitada sobre subastas experimentales con flores, específicamente rosas, y ayudarían a los productores locales a establecer estrategias adecuadas para desarrollar la futura demanda de estos productos.

El documento está estructurado en cuatro secciones adicionales. La primera sección describe los antecedentes. La segunda, se enfoca en el marco metodológico. La tercera sección está dedicada a los resultados y, por último, el documento finaliza con algunas conclusiones. 


\section{REVISION DE LITERATURA}

La calidad es un concepto complejo y multidimensional. Es complicado diferenciar la calidad debido a los enfoques que se pueden tomar para analizarla. Son dos los enfoques principales: objetivo y subjetivo. En términos generales, la calidad para los productores se basa en la medida de estándares con los objetivos que un producto debe cumplir específicamente, toma parámetros para medir los estándares; y en este sentido la calidad es objetiva. Por otro lado, la calidad en algún momento se refiere a la percepción del consumidor, los consumidores utilizan un lenguaje vago para definir lo que significa para ellos calidad. Además, presentan una extraña actitud analítica al momento de adquirir un bien o servicio, es en ese momento que el concepto de calidad es un término subjetivo (Lawless, 1995).

En la evaluación del concepto de calidad, desde una perspectiva objetiva autores como Crosby (1979) citado en Hoyer y Hoyer (2001) lo define como el cumplimiento de los requisitos, que deben establecerse claramente para que no puedan interpretarse erróneamente, entonces, la calidad es el cumplimiento de los requisitos establecidos. Juran (1988) citado en Hoyer y Hoyer (2001) define la calidad como adecuación al uso, donde las características del producto conducen a la satisfacción del producto, por lo tanto, está libre de deficiencias. Ambos autores definen la calidad con base a las características físicas de un producto, la cuales se puede medir, y, por lo tanto, la calidad en este sentido es objetiva. Para el mercado florícola mexicano una de las principales características de la calidad es la inexistencia de índices de calidad, sólo se tienen en cuenta ciertas variables que determinan significativamente la calidad de las flores de corte.

La segunda perspectiva en la que se evalúa la calidad, de acuerdo con Feigenbaum (1983) citado en Hoyer y Hoyer (2001), la calidad es determinada por los consumidores, en función de la experiencia con el producto o servicio, medido frente a los requisitos y/ o expectativas; de lo cual se puede inferir que la calidad es totalmente subjetiva y es percibida por los sentidos, y esto la hace multidimensional. La calidad en algún momento se refiere a la percepción del consumidor, sin embargo, la percepción del consumidor no es estática a lo largo del tiempo o estable entre diferentes tipos de consumidores, y la idea sobre calidad de los consumidores cambia con la experiencia (Lawless, 1995). La calidad subjetiva está orientada al consumidor, es dinámica y multidimensional. Es una combinación de las características que afectan el grado de aceptación por parte de los consumidores, convirtiéndolos en los evaluadores finales en el mercado. De ahí la importancia de tomar la opinión de los consumidores como punto de referencia, ya que la disposición a pagar por un bien o servicio depende de su evaluación; a través de la relación calidad-precio, que en cierta medida determina el valor o utilidad percibida por los consumidores.

Durante la última década, se ha cuestionado la importancia de los resultados obtenidos a partir de métodos de valoración hipotética. Se ha observado que los consumidores no revelan su comportamiento económico real por medio de preguntas de valoración hipotéticas, sobrestimando su disposición real a pagar, en contraste con lo que sucede en experimentos con incentivos económicos reales (List y Gallet, 2001; Lusk y Schroeder, 2004; Lusk y Schroeder, 2006). El hecho de que el dinero real se intercambie en métodos 
de valoración experimentales puede hacer que los participantes sean más receptivos a revelar su verdadero valor para un producto en comparación con la configuración hipotética de la encuesta. Las subastas experimentales se definen como una institución de mercado para determinar los precios y asignar las mercancías. Una subasta se define como una institución de mercado que tiene un conjunto explícito de reglas que determinan la asignación de recursos y donde los precios se basan en las ofertas presentadas por los participantes (Momparler y Hidalgo, 2005). Es un método para obtener el análisis costo-beneficio, obteniendo los valores de bienes reales. En otras palabras, se estima lo que el consumidor está dispuesto a pagar.

Una de las principales ventajas de las subastas experimentales es que ubican a los sujetos en un entorno de mercado activo donde pueden aprender y adaptarse a las condiciones del mercado. Por otra parte, las ofertas proporcionan a los investigadores una estimación explícita para la DAP de cada participante sin la necesidad de estimar un modelo econométrico. Además, la subasta experimental permite predecir el éxito de nuevos productos en el mercado y medir el éxito futuro de los productos ya existentes; con el propósito de entender el comportamiento de los consumidores en un grupo y de una manera independiente. La disposición a pagar expresa la cantidad máxima que un consumidor pagaría para adquirir un bien. Según Villalobos y Wolff (2002), es la valoración personal de ese bien, el cual luego se convierte en una medida subjetiva debido a que es diferente para cada consumidor.

Las transformaciones demográficas, socioculturales y el comportamiento de los consumidores, junto con el desarrollo de nuevas preferencias por los bienes innovadores y la aparición de nuevos segmentos de consumidores, cada vez más específicos en gustos y preferencias hacen que los atributos de calidad del producto sean un factor primordial a la hora de comprar (Berges y Casella, 2008). Berges y Casella (2008) también sugieren que, aunque los consumidores están preocupados por la calidad de los productos que consumen, la disposición a pagar por atributos adicionales es baja. Esta incrementa en relación directa con la información que procesan y si dan prioridad a las cuestiones de calidad y seguridad antes que el precio, a la hora de decidir sus compras.

El análisis sensorial consiste en la realización de diversas pruebas para evaluar diferentes propiedades o atributos de un producto utilizando los sentidos. La percepción sensorial juega un papel decisivo en la caracterización y evaluación de los productos, en este caso no solo intervienen los cinco sentidos, sino también el factor afectivo, es decir, aceptación o rechazo; e influye en la percepción de calidad de los consumidores y, por lo tanto, en la disposición a pagar que cada consumidor genera hacia el producto evaluado. El propósito de los productos es su consumo. Además de su uso final, las propiedades sensoriales son muy importantes en la aceptación de los productos por parte de los consumidores. Desde este punto de vista, es una herramienta importante para el desarrollo de nuevos productos (CSIC-UAM, 2011). El objetivo del análisis sensorial es establecer una calidad básica del producto, que verifique la ausencia o presencia de defectos en el producto a través de una evaluación organoléptica. 
Figura 1. Etapas del análisis sensorial.

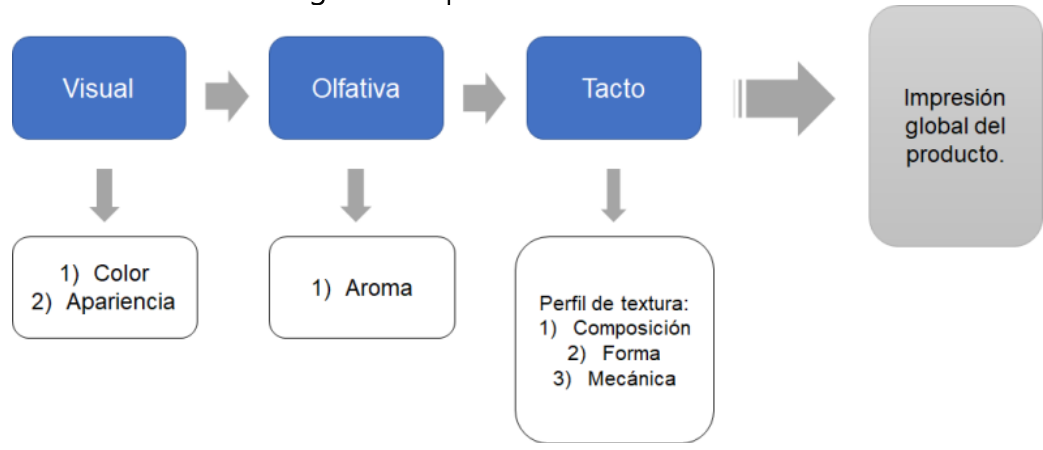

Fuente: Elaboración propia con base en CSIC-UAM (2011).

La Figura 1 ilustra los pasos del análisis sensorial que se llevaron a cabo, comenzando con la fase visual donde se evalúa el color y la apariencia de la rosa, luego evaluando el aroma a través de la fase olfativa y finalmente el tacto, donde la frescura de la flor es calificada; para obtener una visión general del producto evaluado. Para el propósito de esta investigación, la subasta de la oferta del segundo precio fue elegida, llamada también subastas Vickrey. En este tipo de subasta, los compradores potenciales presentan la oferta en un sobre cerrado, en un formato previamente establecido por el vendedor. El bien se otorga al mejor postor y el precio a pagar es la segunda oferta más alta. En el momento en que se presentan las ofertas, los compradores no saben cuáles son las otras ofertas y cada postor puede presentar una sola oferta. En este sentido, cada comprador debe adivinar el comportamiento de los demás para finalmente ofrecer el valor real que representa el producto (Momparler e Hidalgo, 2005).

El hecho de que la veracidad sea una estrategia dominante también hace que las subastas de segundo precio sean conceptualmente más limpias. Debido a que la oferta veraz es una estrategia dominante, es lo mejor que se puede hacer sin importar lo que hagan los otros postores. Por lo tanto, en una subasta de segundo precio, tiene sentido ofrecer su verdadero valor incluso si otros postores son excesivos, ofertan menos de lo que está dispuesto a pagar, coludir o comportarse de otras maneras impredecibles. En otras palabras, una oferta veraz es una buena idea (Easley y Kleinberg, 2010).

\section{METODOLOGÍA}

\section{Datos}

Para este estudio la muestra de población fue seleccionada de Toluca, Estado de México, por ser la ciudad más grande y cercana a la zona de producción de flores, y de área metropolitana. Los participantes fueron reclutados vía teléfono, por invitación y volantes para participar en un estudio de preferencia de rosa de corte. El único criterio que se estableció para la selección fue que los participantes fueran mayores de 18 años. Ocho sesiones de 14 participantes y dos sesiones de 10 participantes se llevaron a cabo en noviembre de 2016. En donde 122 personas participaron, de las cuales 22 fueron invalidadas por falta de datos.

No se otorgó ningún incentivo económico a los participantes. La tabla 1 muestra las principales características de los participantes y la tabla 2 muestra la distribución poblacional de Toluca. La muestra estaba compuesta por un 41\% de hombres y un 59\% 
de mujeres. El 65\% de los participantes tenían entre 18 y 42 años. La mayoría de la muestra (más del 90\%) había terminado la escuela secundaria y revelaron tener un ingreso entre 45.11 y 947.38 dólares americanos por mes.

Tabla 1. Distribución demográfica de la muestra

\begin{tabular}{lc}
\hline Variable demográfica & $N=100$ \\
\hline Género & \\
\hline Hombre & $41 \%$ \\
Mujer & $59 \%$ \\
\hline Edad & $26 \%$ \\
\hline $18-26$ & $20 \%$ \\
$27-34$ & $19 \%$ \\
$35-42$ & $12 \%$ \\
$43-50$ & $23 \%$ \\
Más de 51 & \\
\hline Nivel educativo & $1 \%$ \\
\hline Educación básica sin terminar & $3 \%$ \\
Educación básica terminada & $5 \%$ \\
Educación media superior sin terminar & $15 \%$ \\
Educación media superior terminada & $53 \%$ \\
Estudios universitarios terminados & $23 \%$ \\
Estudios de posgrado & \\
\hline Ingreso & $12 \%$ \\
\hline 46.55 USD - 390 USD & $24 \%$ \\
391 USD - 733 USD & $16 \%$ \\
734 USD - 1,077 USD & $9 \%$ \\
1,078 USD - 1,419 USD & $4 \%$ \\
1,420 USD - 1,763 USD & $1 \%$ \\
1,764 USD - 2,107 USD & $3 \%$ \\
Más de 2,108 USD & $31 \%$ \\
N/A &
\end{tabular}

Tabla 2. Distribución demográfica de Toluca

\begin{tabular}{cc}
\hline Variable demográfica & $\mathrm{N}=819,561$ \\
\hline Género & \\
Hombre & $48.18 \%$ \\
Mujer & $51.82 \%$ \\
\hline Edad & $28.11 \%$ \\
\hline $0-14$ & $18.55 \%$ \\
$15-24$ & $16.45 \%$ \\
$25-34$ & $14.79 \%$ \\
$35-44$ & $5.53 \%$ \\
$45-49$ & $16.57 \%$ \\
\hline Más de 50 & Datos no disponibles \\
\hline Nivel educativo & Datos no disponibles \\
\hline Fuente: INEGI, Censo de Población y Vivienda, 2010. Estados Unidos \\
Mexicanos y Estado de México, Tabulados del cuestionario Básico, \\
Población.
\end{tabular}




\section{Procedimiento experimental}

Se realizó un análisis sensorial para determinar la percepción de calidad de los consumidores y una subasta experimental (subasta de Vickrey) con el objetivo de encontrar la disposición a pagar de los consumidores de rosa de corte del sur del Estado de México. El experimento se realizó en tres etapas: 1) Bienvenida e introducción al experimento; 2) análisis sensorial y 3) subasta de Vickrey. Durante la etapa introductoria, cada encuestado fue asignado en un espacio separado. Posteriormente, se realizó una breve explicación sobre los objetivos del experimento y la confidencialidad en la obtención de los datos. Además, se pidió a los participantes que se comportaran como en la vida real.

Etapa 1: Se les mostraron a los participantes tres tipos de rosas al azar de diferentes calidades: calidad estándar, nacional y de exportación.

Etapa 2: Los participantes evaluaron mediante un análisis sensorial la calidad de cada muestra. En esta etapa, se les proporciono un formato con 7 preguntas para evaluar diferentes atributos relacionados con la calidad de la rosa (olor, aroma y color). Se usó la escala Likert (0-7) para evaluar dichos atributos, donde 0 fue "No me gusta" y 7 fue "Me gusta totalmente".

Etapa 3: El último paso consistió en la subasta de Vickrey. Las ofertas tenían que ser el precio más alto que estaban dispuestos a pagar por cada una de las muestras de la rosa de corte. Además, a los participantes se les dijo que el ganador tendría que pagar el segundo precio por la rosa que se seleccionaría al azar.

\section{Procedimiento analítico}

El método de regresión se utiliza en el campo de las ciencias sociales y experimentales, se esfuerza por conocer la relación entre las variables. La aplicación de este método busca modelar esta relación para su uso predictivo o análisis estructural. En la regresión cuantílica, el objetivo es minimizar una suma de errores absolutos ponderados asimétricos (Vicéns y Sánchez, 2012). La regresión cuantílica se usa cuando se presentan heterocedasticidad, cambios estructurales o datos atípicos. Es decir, la información no se puede adaptar a otro método más simple. La regresión cuantil ofrece la posibilidad de crear diferentes líneas de regresión para un cuantil diferente de la variable endógena, a través de un método de estimación que se ve menos afectado por tales desventajas. La principal ventaja de la regresión cuantílica en esta estrategia es que en cada cuantil todas las observaciones se ponderan convenientemente.

Como antecedente, se consideró la forma tradicional de un modelo de regresión condicional. Los parámetros que se estiman en una regresión tradicional para los parámetros elegidos, $\beta$, que minimizan la suma de los errores cuadrados: minimizar ( $y_{i}$ $\left.-X_{i} \beta\right)$, donde " $\mathrm{y}$ " es la variable dependiente $y$ " $X$ " es un conjunto de variables independientes. En una regresión cuantílica, la suma de los errores cuadrados se reemplaza con la función objetivo (Lusk y Shogren, 2007).

1 En primer lugar, se realizó un experimento piloto. Su objetivo era probar el procedimiento experimental. Se empleó un total de dos grupos de 10 participantes (estudiantes). 


$$
\text { Minimizar } \sum_{i=1}^{N} \rho \theta(Y i-X i \beta)
$$

Donde $\theta$ es el cuantil y $\rho$ es una función de valor absoluto ponderado (también conocida como función de "verificación") definida de la siguiente manera:

$$
\rho \theta(y i-X i \beta)=\left\{\begin{array}{c|c}
\theta(Y i-X i \beta) & \begin{array}{l}
\text { si } Y i-X i \beta>0 \\
\text { si } Y i-X i \beta \leq 0
\end{array}
\end{array}\right\}
$$

\section{RESULTADOS}

Los resultados del experimento se presentan a continuación. La tabla 3 corresponde a la prueba de medias donde se observa cómo los consumidores modifican la percepción de calidad hacia la rosa de corte después del análisis sensorial de este producto.

Tabla 3. Prueba de media para las muestras de rosa calidad estándar, nacional y exportación.

\begin{tabular}{lllllll}
\hline Muestra & \multicolumn{2}{l}{ Rosa calidad nacional } & \multicolumn{2}{l}{ Rosa calidad estándar } & \multicolumn{2}{l}{ Rosa calidad exportación } \\
\hline Análisis sensorial & Antes & Después & Antes & Después & Antes & Después \\
\hline Promedio & 5.67 & 5.47 & 4.38 & 4.02 & 4.62 & 4.36 \\
Error estándar & 0.12 & 0.13 & 0.17 & 0.17 & 0.16 & 0.17 \\
Desviación estándar & 1.25 & 1.29 & 1.67 & 1.75 & 1.63 & 1.68 \\
\hline T & 2.35 & & 3.43 & & 2.91 & \\
\hline P & 0.021 & 0.001 & & 0.005 & \\
\hline
\end{tabular}

A continuación, se muestra que el consumidor es consistente al declarar si a la persona le gusta la rosa antes y después del análisis sensorial para los tres tipos de calidad de rosa. Aunque debe tenerse en cuenta que hay una ligera reconsideración de su apreciación después de un análisis sensorial (Figuras 2a; 2b; 2c).

\section{Figura 2a. Consistencia en la preferencia del consumidor (rosa nacional)}

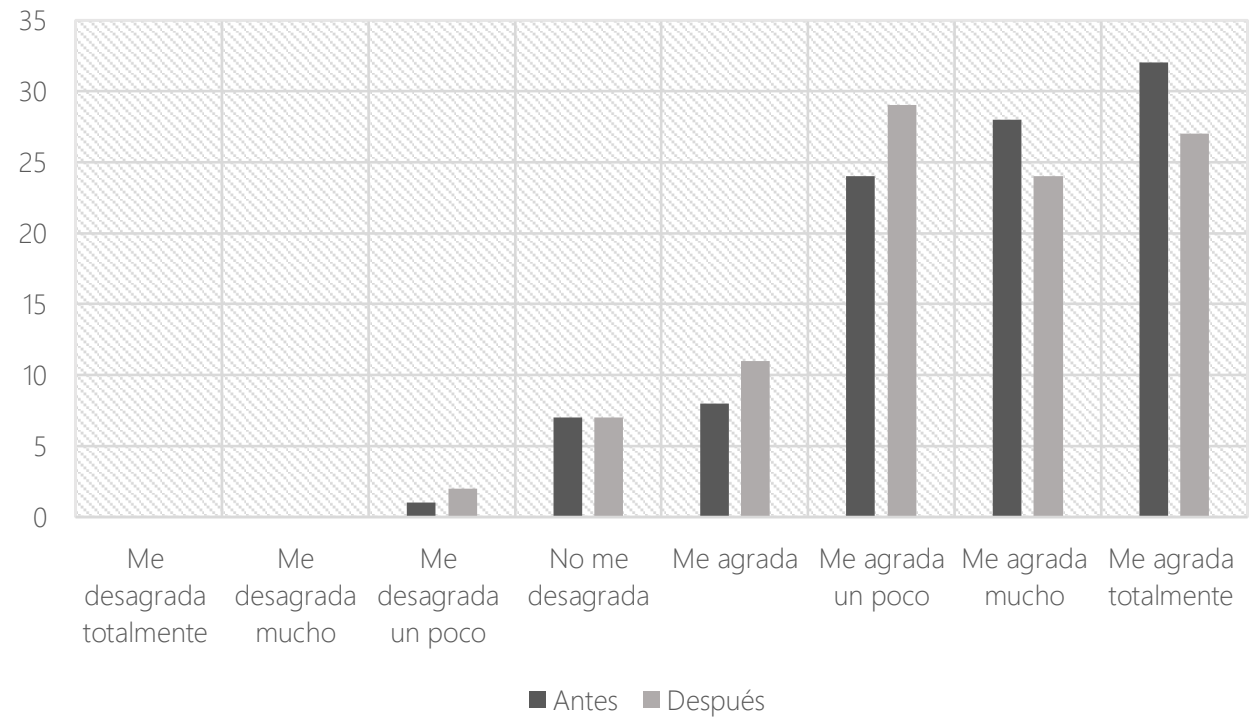


Figura 2b. Consistencia en la preferencia del consumidor (rosa estándar)

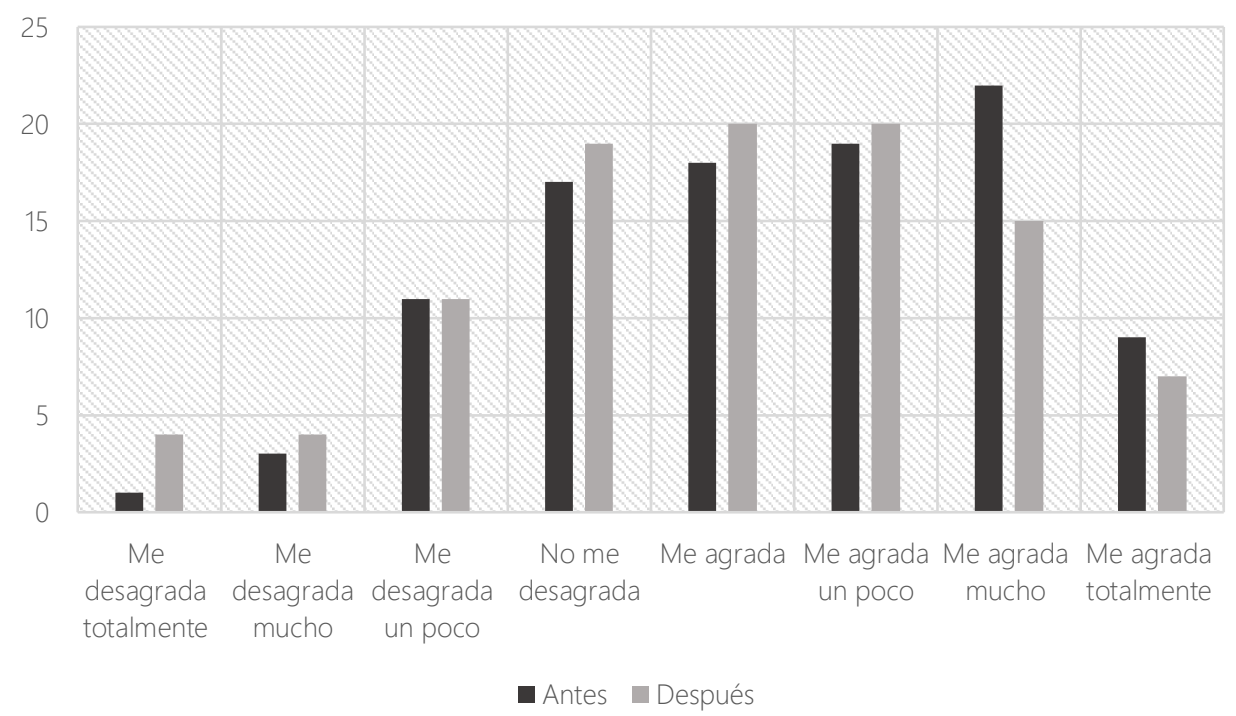

Figura 2c. Consistencia en la preferencia del consumidor (rosa de exportación)

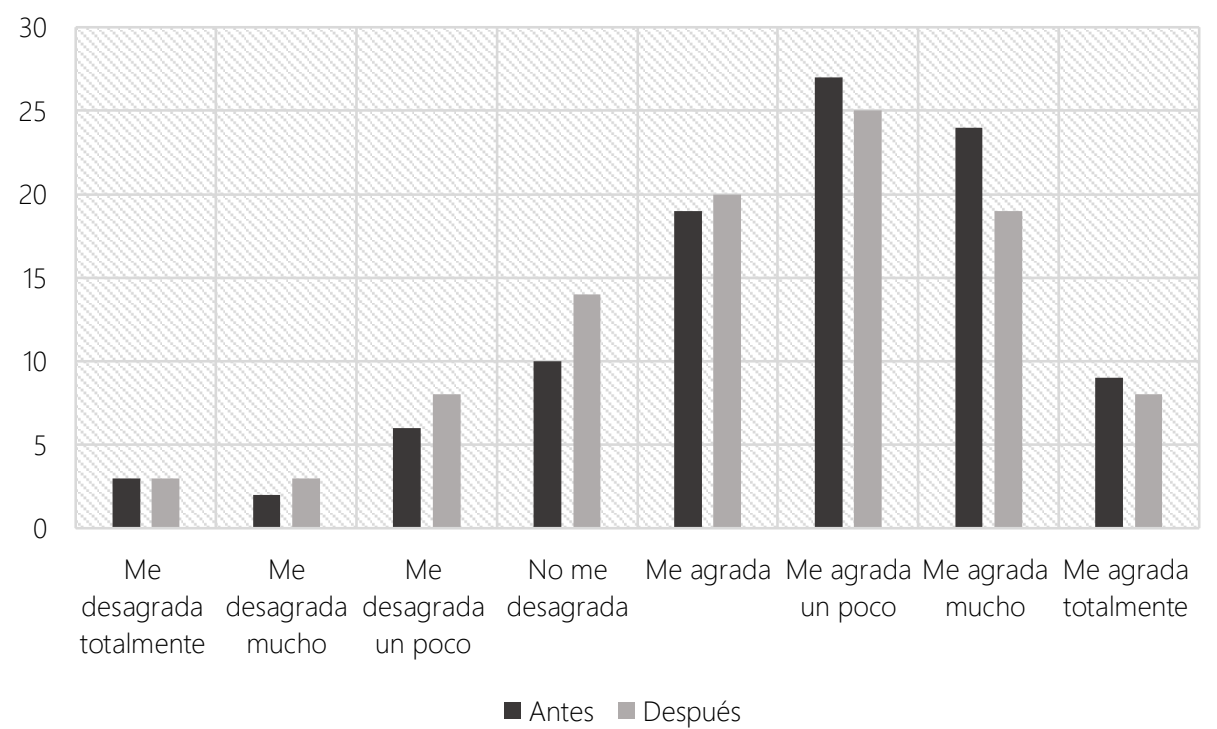

La figura 3 muestra que el 75\% de los consumidores declaran que la rosa nacional es una rosa de calidad, mientras que un poco más del $50 \%$ consideran que la rosa estándar no es una rosa de calidad. Finalmente, un poco más del 50\% de los consumidores dice que la rosa de exportación es una rosa de calidad. 
Figura 3. Percepción de calidad.

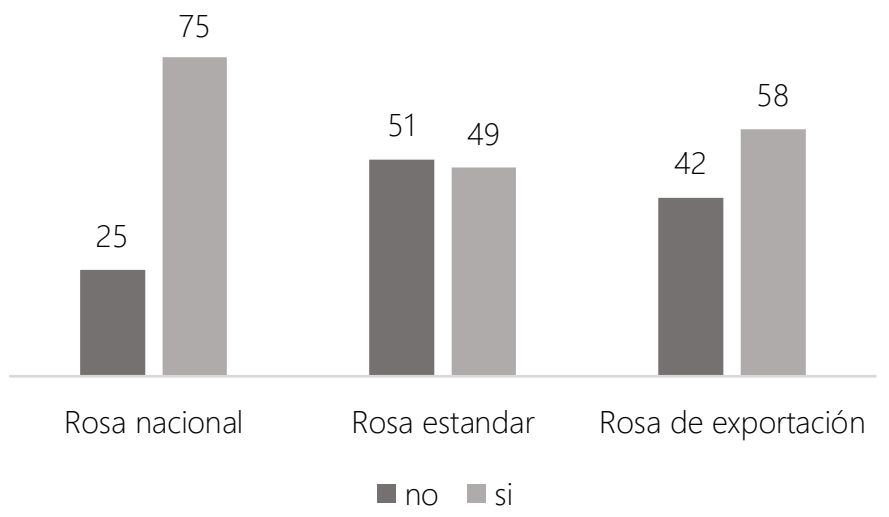

La figura 4 indica que el precio promedio que los consumidores están dispuestos a pagar por el aumento de calidad nacional es de 10 pesos mexicanos.

Figura 4. Frecuencia de precios para la subasta de la rosa nacional.

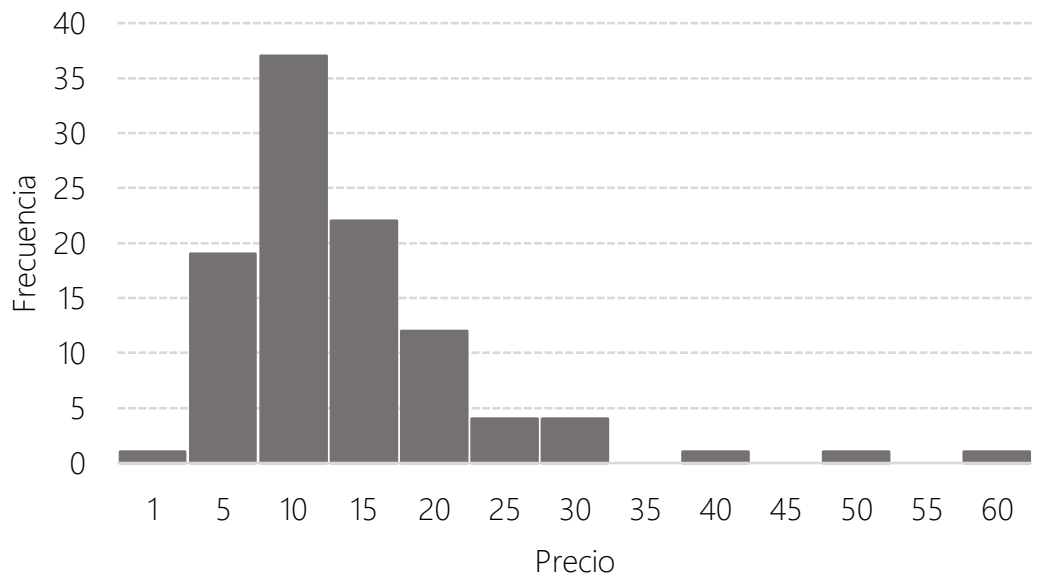

La figura 5 muestra que la mayoría de los consumidores están dispuestos a pagar 5 pesos mexicanos por la rosa de calidad estándar.

Figura 5. Frecuencia de precios para la subasta de rosas estándar

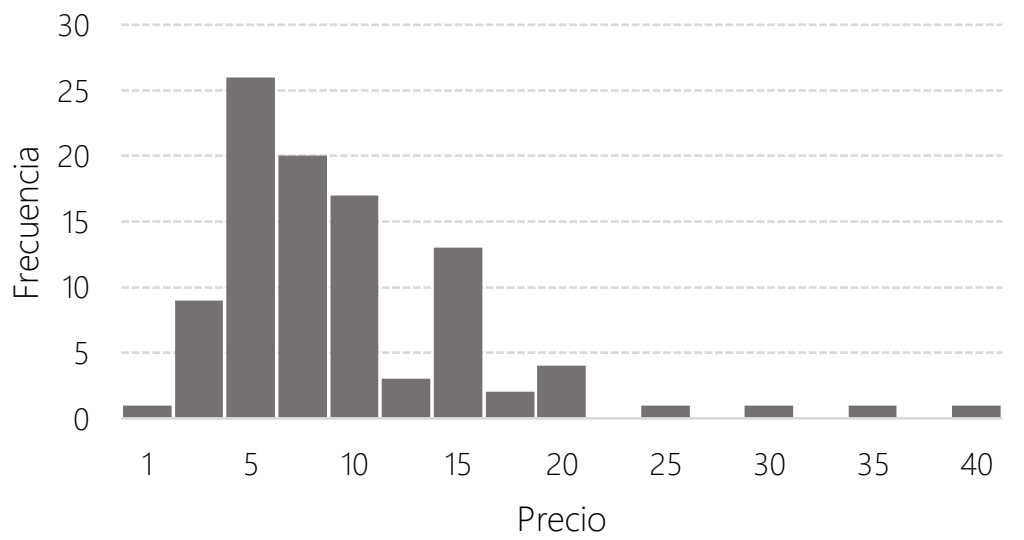


La figura 6 muestra que, al igual que con la rosa de calidad nacional, los consumidores están dispuestos a pagar un promedio de \$10 MXN por rosa con calidad de exportación.

Figura 6. Frecuencia de precios para la subasta de rosa de exportación.

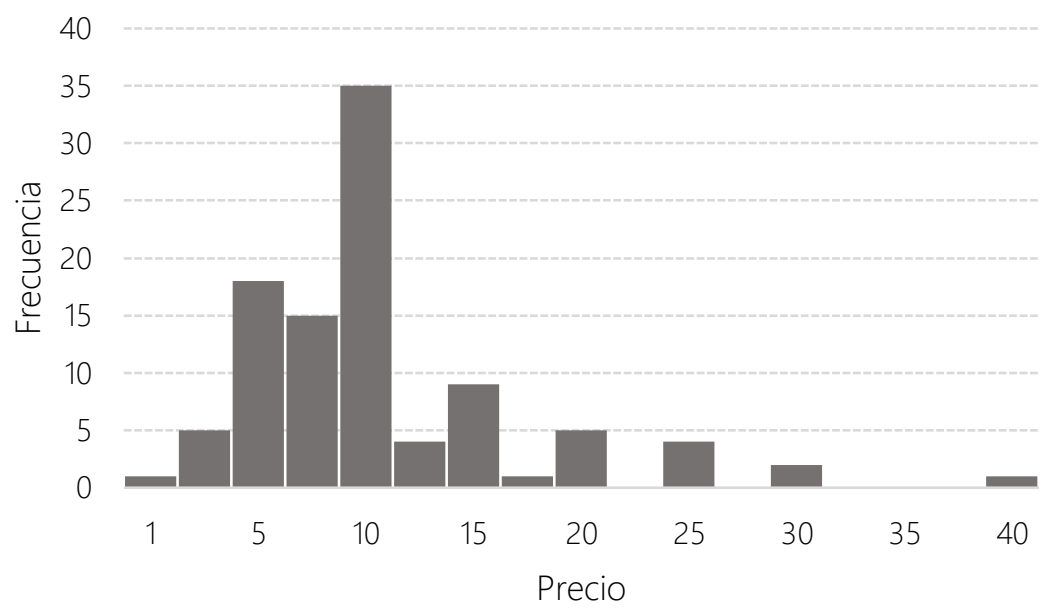

En la tabla 4 se observa que el precio promedio de la rosa nacional es de $\$ 13.7$ pesos mexicanos; mientras que para la rosa estándar es de $\$ 9.6$ y para la rosa de exportación el precio promedio es de $\$ 10.78$ pesos mexicanos.

Tabla 4. Estadísticas descriptivas para las subastas de los diferentes tipos de rosas (MXN)

\begin{tabular}{lccc} 
& $\begin{array}{c}\text { Subasta rosa } \\
\text { nacional }\end{array}$ & $\begin{array}{c}\text { Subasta rosa } \\
\text { estándar }\end{array}$ & $\begin{array}{c}\text { Subasta rosa de } \\
\text { exportación }\end{array}$ \\
\hline Media & 13.7 & 9.6 & 10.78 \\
Error estándar & 0.92 & 0.67 & 0.66 \\
Mediana & 10 & 8 & 10 \\
Moda & 10 & 5 & 10 \\
Desviación estándar & 9.23 & 6.66 & 6.63 \\
\hline
\end{tabular}

La tabla 5 muestra los precios que tiene la rosa en el mercado de acuerdo con su clasificación. También muestra el precio que los consumidores están dispuestos a pagar por cada una de las rosas. Se observa que por la rosa considerada como de menor calidad (según los productores) los consumidores están dispuestos a pagar 27 veces más que el valor de mercado. Mientras que, para la rosa estándar, los consumidores están dispuestos a pagar un poco menos de 3 veces más y para el caso de la rosa de calidad de exportación, el consumidor está dispuesto a pagar solamente 78 centavos de peso más que el valor de mercado.

Tabla 5. Precios en el mercado para diferentes clasificaciones de rosa (MXN)

\begin{tabular}{lccc}
\hline Clasificación de la rosa & $\begin{array}{c}\text { Precio de } \\
\text { mercado por } \\
\text { paquete }\end{array}$ & $\begin{array}{c}\text { Precio de } \\
\text { mercado } \\
\text { por pieza }\end{array}$ & $\begin{array}{c}\text { Precio declarado } \\
\text { por el consumidos } \\
\text { por pieza }\end{array}$ \\
\hline Rosa nacional & $\$ 50.00$ & $\$ 0.50$ & $\$ 13.70$ \\
Rosa estándar & $\$ 90.00$ & $\$ 3.60$ & $\$ 9.60$ \\
Rosa de exportación & $\$ 205.00$ & $\$ 10.00$ & $\$ 10.78$ \\
\hline
\end{tabular}


Tanto las tablas como las cifras de frecuencia de precios indican que los consumidores están dispuestos a pagar más por la rosa nacional que por la rosa estándar o de exportación. De acuerdo con la clasificación utilizada por los productores, que se adhieren a los estándares de calidad, la rosa nacional es la rosa de menor calidad. Sin embargo, los consumidores están dispuestos a pagar hasta 27 veces más, debido a que los consumidores consideran que esta rosa es la de mejor calidad. Es interesante observar que la disponibilidad del pago del consumidor no está relacionada en su totalidad con los índices de calidad establecidos en las políticas oficiales mexicanas para la rosa de corte; lo que se traduce en una brecha entre lo que el productor ofrece en el mercado y lo que el consumidor realmente percibe de calidad a través de los atributos físicos de la rosa.

La tabla 6 muestra los resultados para cada cuantil realizado tomando como variable dependiente la disponibilidad de pago; la cual explica la relación entre la disposición a pagar de las rosas y las variables sociodemográficas (ingresos, género, edad y situación laboral); así como su relación con la calidad para cada rosa desde la percepción del consumidor.

Tabla 6. Resultados de las estimaciones

\begin{tabular}{|c|c|c|c|}
\hline $\begin{array}{c}\text { Ecuación } \\
\text { DAP }\end{array}$ & Cuantil 25 & Cuantil 50 & Cuantil 75 \\
\hline Ingreso & $0.000^{* \star}(3.86)$ & $0.092 *(1.17)$ & $0.544(0.61)$ \\
\hline $\begin{array}{l}\text { ¿Le gusta esta rosa? } \\
\text { (Rosa Nacional) }\end{array}$ & $0.725(-0.35)$ & $0.901(0.13)$ & $0.422(0.81)$ \\
\hline $\begin{array}{l}\text { ¿Es una rosa de calidad? } \\
\text { (Rosa Nacional) }\end{array}$ & $0.047^{\star \star}(2.02)$ & $0.527(0.64)$ & $0.686(-0.41)$ \\
\hline $\begin{array}{l}\text { ¿Le gusta esta rosa? } \\
\text { (Rosa Estándar) }\end{array}$ & $0.462(0.74)$ & $0.129(1.53)$ & $0.321(1.00)$ \\
\hline $\begin{array}{l}\text { ¿Es una rosa de calidad? } \\
\text { (Rosa Estándar) }\end{array}$ & $0.921(-0.10)$ & $0.054^{*}(-1.95)$ & $0.22(-1.23)$ \\
\hline $\begin{array}{l}\text { ¿Le gusta esta rosa? } \\
\text { (Rosa de Exportación) }\end{array}$ & $0.799(0.26)$ & $0.580(-0.56)$ & $0.196(-1.30)$ \\
\hline $\begin{array}{l}\text { ¿Es una rosa de calidad? } \\
\text { (Rosa de Exportación) }\end{array}$ & $0.098 *(1.67)$ & $0.025^{\star \star}(2.28)$ & $0.055^{*}(1.95)$ \\
\hline Género & $0.061^{*}(1.90)$ & $0.142(1.48)$ & $0.606(0.52)$ \\
\hline Edad & $0.046^{* *}(-2.02)$ & $0.721(-0.36)$ & $0.607(-.043)$ \\
\hline Situación laboral & $0.000 * *(-3.92)$ & $0.119(-1.57)$ & $0.292(-1.06)$ \\
\hline Contras & $0.015^{\star *}(2.48)$ & $0.721(0.36)$ & $0.726(0.35)$ \\
\hline
\end{tabular}

Estadístico t en paréntesis; *Significativo al 10\%; **5\%.

Para el cuantil 25, el ingreso, el género, la edad y la situación laboral son importantes en la DAP. Además, para este primer cuantil, se encuentra que la DAP está relacionada con la calidad de las rosas nacionales y de exportación. Para el segundo cuantil (50), se observa que los ingresos y la calidad de la rosa nacional y de exportación son significativos. El último cuantil (75) muestra que la calidad de las rosas de exportación es significativa en la DAP.

\section{DISCUSION Y CONCLUSIONES}

De acuerdo con los resultados obtenidos se concluye que la disposición a pagar (DAP) está vinculada a los ingresos y la percepción de calidad que los consumidores tienen sobre las rosas. A medida que la economía de un país mejora, la demanda de flores 
aumenta, ya que este es considerado como un producto de lujo, y es importante considerar el factor ingreso. Se ha demostrado que la segmentación de los consumidores es clave para la venta de rosa de corte (Avitia et al., 2015; Berges y Casellas, 2008). La estimación empírica indica que el ingreso, la calidad y algunas otras variables sociodemográficas (edad, género y situación laboral) están relacionadas con la disposición a pagar.

Se puede concluir que la crisis económica que se extiende por el país explica en parte la baja demanda de rosa. Sin embargo, se observa que los índices de calidad de rosa establecidos por las agencias gubernamentales y los productores de rosas no convergen con el precio que los consumidores están dispuestos a pagar por las diferentes variedades de rosas. En este sentido, los productores deben enfocarse en las características físicas y morfológicas que deben cubrirse en las rosas al momento de la producción junto con las que los consumidores desean y evalúan como calidad en la rosa de corte.

En cuanto a las variables sociodemográficas y su importancia en la disposición a pagar, se pueden sugerir estrategias de segmentación para estimular la demanda de la rosa de corte. La percepción de calidad hacia el corte rosa por parte de los consumidores cambia, después del análisis sensorial de este producto. Por tanto, los consumidores también se agrupan de manera diferente, después de la evaluación sensorial. Los resultados de la investigación permiten al sector florícola de la región (productores, floristas, distribuidores) obtener una mejor comprensión de lo que los consumidores buscan; y así, establecer estrategias con la finalidad de incentivar el consumo del producto.

\section{REFERENCIAS}

Anderson, D., Sweeney, D., \& Williams, T. (1999). Estadística para administración y economía. México: International Thomson Editores.

Avitia, J., Nervis, D., \& Ramírez, J. (2015). Generation X and Generation Y. An approach of consumption values toward roses in the South of the State of Mexico. Milan, Italy: International Conference of Agricultural Economists.

Berges, M., \& Casellas, K. (2008). Disposición a pagar por atributos de calidad. Un estudio aplicado al agregado de nutrientes en la leche fluida. Argentina: Asociación Argentina de Economía Política.

CSIC-UAM. (2011). Curso de análisis sensorial de alimentos. Instituto de Investigación en Ciencias de la Alimentación (CIAL) Instituto Mixto CSIC-UAM.

Easley, D., \& Kleinberg, J. (2010). Networks, Crowds, and Markets: Reasoning about a Highly Connected World. Cambridge University Press.

Economía, S. d. (2002). Productos Hortícolas- Flores cortadas en estado freso- Rosa (Rosa spp.)Especificaciones y método de prueba. México: NMX-FF-069-SCFI-2002.

Gobierno de Chiapas, F. E. (2005). Plan rector sistema producto flores de Chiapas. 1-96.

Grunet, K. (1995). Food quality: a means-end perspective. Food Quality and Preferences, 171-176. Hoyer, R., \& Hoyer, B. (2001). What is quality? Quality Progress, 53-62.

Juárez, P., Colinas, M. T., Valdez, L., Espinosa, A., Castro, R., \& Cano, G. (2008). Soluciones y refrigeración para alargar la vida postcosecha de rosa CV. "Black Magic". Revista Fitotecnia Mexicana, 73-77.

Lawless, H. (1995). Dimensions of sensory quality: a critique. Food quality and preference, 1191-1999.

Lusk, J., \& Shogren, J. (2007). Experimental Auctions: Methods and Applications in Economic and Marketing Research. New York: Cambridge University Press. 
Momparler, J., \& Hidalgo, M. (2005). Modelos de subastas y su aplicación a concursos. Coruña: XIII Jornadasde ASEPUMA.

Prince, T. L. (2015). Consumer perceptions of floral quality and price and their impact on satisfaction/purchasing loyalty: Part I, consumer attribute scores. Prince \& Prince, 2-18.

SIAP. (2014). Servicio de Información Agroalimentaria y Pesquera. México.

Sloof, M., Tijskens, \& Wilkinson. (1996). Concepts for modelling the quality of perishable products. Trends in food science \& technology, 195-171.

Vicéns, J., \& Sánchez, B. (2012). Regresión Cuantílica: Estimación y contrastes. Madrid: Instituto L.R. Klein-Centro Gauss. 